\title{
Reply to "Association of HLA-B27 and its subtypes with ankylosing spondylitis and clinical manifestations of ankylosing spondylitis in different HLA-B27 subtypes: comment on the article by Lin et al."
}

\author{
Hai $\operatorname{Lin}^{1} \cdot$ Yi-Zhen Gong ${ }^{2}$
}

Received: 1 August 2017 / Accepted: 7 August 2017 / Published online: 22 August 2017

(c) Springer-Verlag GmbH Germany 2017

Firstly, this letter mentions we have missed three articles reported by Fallahi et al., Mou et al. and Omair et al. [1-3]. We agree with that we have missed two articles report by Fallahi et al. and Mou et al. [1, 2]. However, we searched eligible studies update to November 2016 and our paper has been online on May 2017. But the study reported by Omair et al. was Epub ahead of print on July, 2017, so we do not agree the point of this letter [3].

Secondly, this letter argues that the missed studies may affect the consequence of our study. After we reviewed these two articles, we do not suggest to include these two studies. In our study, we aimed at exploring the sensitivity of AS patients to HLA-B27, so we only selected studies including AS patients and their control normal groups. As to the relationships of clinical features and HLA-B27 and its subtypes in AS patients, we also follow the same criteria. However, the patients included in these two studies are both AS patients without control groups. And we also find the same problem related to the associations between HLA-B27 subtypes and clinical features in AS patients. Overall, we do not consider these studies to be involved.

Thirdly, this letter also suggests that we should perform subgroup analysis for clinical features of AS patients based

This reply refers to the comment available at doi:10.1007/ s00296-017-3790-6.

Hai Lin

doctor_linhai@163.com

Yi-Zhen Gong

13607887067@163.com

1 Department of Spinal Surgery, The First Affliated Hospital of Guangxi Medical University, Nanning, China

2 Department of Evidence-based Medicine, The First Affliated Hospital of Guangxi Medical University, Nanning, China on different races and ethnicities. We agree to this point and re-analyze the relationship of HLA-B27 and clinical characters in AS patients. Based on different races, the results of our analyses showed that there was a positive relation between HLA-B27 and sex [East Asia: RR $(95 \% \mathrm{CI})=1.05$ $(1.00,1.10), P=0.033$ ], family history [East Asia: RR $(95 \%$ CI) $=1.09(1.05,1.13), P<0.001$; Southwest Asia: RR $(95 \% \mathrm{CI})=1.69(1.30,2.22), P<0.001]$, uveitis [East Asia: RR $(95 \% \mathrm{CI})=1.05(1.01,1.09), P=0.008$; Southwest Asia: RR $(95 \% \mathrm{CI})=1.46(1.08,1.96), P=0.013]$ and peripheral joint involvement [Southwest Asia: RR (95\% CI) $=1.74(1.30,2.34), P<0.001]$. However, as the limitations of eligible studies, it is difficult to conduct subgroup analysis for the relationships of HLA-B27 subtypes and clinical parameters in AS patients. Therefore, more relevant studies should be conducted to identify these results.

Thanks for the advices suggested from this letter.

Best regards,

Dr. Lin.

\section{References}

1. Fallahi S, Mahmoudi M, Nicknam MH, Gharibdoost F, Farhadi E, Saei A, Nourijelyani K, Ahmadzadeh N, Jamshidi AR (2013) Effect of HLA-B*27 and its subtypes on clinical manifestations and severity of ankylosing spondylitis in Iranian patients. Iran $\mathbf{J}$ Allergy Asthma Immunol 12(4):321-330

2. Mou Y, Zhang P, Li Q, Lin Z, Liao Z, Wei Q, Gu J (2015) Clinical features in juvenile-onset ankylosing spondylitis patients carrying different B27 subtypes. Biomed Res Int. doi:10.1155/2015/594878

3. Omair MA, AlDuraibi FK, Bedaiwi MK, Abdulaziz S, Husain W, El Dessougi M, Alhumaidan H, Al Khabbaz HJ, Alahmadi I, Omair MA, Al Saleh S, Alismael K, Al Awwami M (2017) Prevalence of HLA-B27 in the general population and in patients with axial spondyloarthritis in Saudi Arabia. Clin Rheumatol. doi:10.1007/s10067-017-3655-0 (Epub ahead of print) 